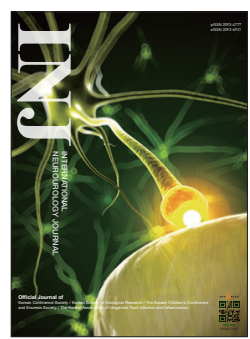

\title{
Current Challenges of Geriatric Voiding Dysfunction in South Korea
}

\author{
Sung Tae Cho (iD http://orcid.org/0000-0002-4691-6159 \\ Department of Urology, Hallym University College of Medicine, Seoul, Korea \\ E-mail: cst326@paran.com
}

Voiding dysfunction, an emerging global public health concern, is a complex disorder that lacks a single all-encompassing definition. The International Continence Society/International Urogynecological Association joint terminology and classification report defines voiding dysfunction as "abnormally slow and/or incomplete micturition," based on the symptomatology and urodynamic investigations [1]. However, the term "voiding dysfunction" is empirically used to describe several conditions in which there is a lack of or poor coordination between the bladder and the urethra during voiding [2]. It includes various conditions such as benign prostatic hyperplasia $(\mathrm{BPH})$, urinary incontinence (UI), overactive bladder (OAB), lower urinary tract symptoms (LUTS), and nocturia, which are prevalent in the elderly population.

The global population, including the Korean population, has been aging considerably since the mid-20th century. Aging is associated with a general decline in almost every physiological function of many biological systems, including the lower urinary tract [3]. The overall prevalence of voiding dysfunction is reported to increase with age in both men and women [4]. Along with the increase in the elderly population, the incidences of UI, LUTS, and OAB have also increased considerably in the Korean population. Therefore, determination of their prevalence rates is an important initial step for epidemiologic characterization of diseases in South Korea.

Choi and Bae [5] analyzed the prevalence of voiding dysfunction through a medical database search using different terms such as BPH, UI, LUTS, OAB, and nocturia. When dis- cussing the epidemiology and impact of voiding dysfunction, it is important to distinguish between its prevalence and incidence. Prevalence, which is the probability of having voiding dysfunction within a defined population at a defined point in time, is a more important statistic compared to incidence, when considering the impact and utilization of healthcare resources [6].

Several data collection methods are used to administer surveys. Different methods of survey data collection create mode effects, and different methods can be used for different parts of the same survey. Traditionally, surveys were conducted via faceto-face interviews, telephone interviews, and mailed questionnaires. However, with the advent of innovative computer- and web-based technologies, the traditional paper-based survey is fast changing to computer-assisted interviewing. Postal surveys, telephone surveys, and face-to-face personal interviews are all rapidly being replaced by mobile or online surveys [7]. These newer methods are becoming increasingly popular and essential research tools for a variety of study fields.

The reported prevalence rates for voiding dysfunction in men and women vary considerably, depending on study designs, the survey and data collection methods used, definitions, and other factors that have been discussed in previous studies [6], where each specific survey method and criterion that defines the clinical conditions of voiding dysfunction has been reviewed in detail. The reported data regarding the prevalence of voiding dysfunction in the Korean population is comparable, albeit with slight differences, to other worldwide reports. This 


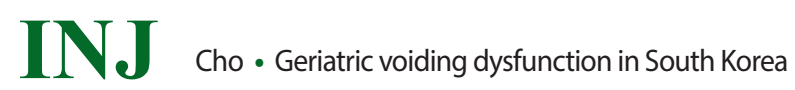

review provides a valuable overview of the epidemiologic studies and methodological issues on voiding dysfunction in the Korean population.

- Conflict of Interest: No potential conflicts of interest relevant to this study exist.

\section{REFERENCES}

1. Haylen BT, Maher CF, Barber MD, Camargo S, Dandolu V, Digesu A, et al. An International Urogynecological Association (IUGA)/ International Continence Society (ICS) Joint Report on the Terminology for Female Pelvic Organ Prolapse (POP). Neurourol Uro- dyn 2016;35:137-68.

2. Karmakar D, Sharma JB. Current concepts in voiding dysfunction and dysfunctional voiding: a review from a urogynaecologist's perspective. J Midlife Health 2014;5:104-10.

3. Siroky MB. The aging bladder. Rev Urol 2004;6(Suppl 1):S3-7.

4. Jung HB, Kim HJ, Cho ST. A current perspective on geriatric lower urinary tract dysfunction. Korean J Urol 2015;56:266-75.

5. Choi H, Bae JH. Overview of epidemiology on voiding dysfunction in Korea. Int Neurourol J 2016;20:91-100.

6. Nitti VW. The prevalence of urinary incontinence. Rev Urol 2001;3 Suppl 1:S2-6.

7. Bethlehem JG, Biffignandi S, editors. Handbook of web surveys. Hoboken (NJ): Wiley; 2012. 